\title{
Tranylcypromine, a lysine-specific demethylase 1 (LSD1) inhibitor, suppresses lesion growth and improves generalized hyperalgesia in mouse with induced endometriosis
}

\author{
Qunyan Sun ${ }^{1+}$, Ding Ding ${ }^{2+}$, Xishi Liu ${ }^{2,3+}$ and Sun-Wei Guo $2,3^{*}$
}

\begin{abstract}
Background: Growing evidence indicates that endometriosis is an epigenetic disease. Encouragingly, histone deacetylases (HDACs) and DNA methyltransferases have been shown to be promising targets by numerous in vitro studies. However, only a few studies have shown promising effects of HDAC inhibition in preclinical studies in endometriosis. While lysine-specific demethylase 1 (LSD1) is recently found to be aberrantly expressed in endometriosis, and that the treatment of endometriotic stromal cells with tranylcypromine (TC), an LSD1 inhibitor, significantly reduced cellular proliferation, cell cycle progression, and invasiveness, the in vivo effect of TC treatment is currently lacking. This study sought to evaluate the effect of TC in a mouse model of endometriosis.
\end{abstract}

Methods: Forty-seven female C57BL/6 mice were used in this experimentation. All mice, except those randomly selected to form Sham surgery (M) and specificity control (S) groups, received an endometriosis-inducing surgery. Group S was set up mainly to ensure that the reduced generalized hyperalgesia in mice treated with TC is not due to any possible analgesic effect of $\mathrm{TC}$, but rather resulting from the treatment effect specific to endometriosis. Two weeks after the surgery, mice that received surgery were further divided randomly into 3 groups: 1) untreated group (U); 2) low-dose TC group (L); 3) high-dose TC group (H). Group $S$ received the same treatment as in group H. Two weeks after treatment, all mice were sacrificed and their ectopic endometrial tissues were harvested and analyzed by immunohistochemistry analysis. Hotplate test was administrated to all mice before the induction, treatment and sacrifice. Lesion size, hotplate latency, immunoreactivity against markers of proliferation, angiogenesis, H3K4 methylation, and of epithelial-mesenchymal transition (EMT).

Results: TC treatment significantly and substantially reduced the lesion size and improved generalized hyperalgesia in a dose-dependent fashion in mice with induced endometriosis. In addition, TC treatment resulted in reduced immunoreactivity to biomarkers of proliferation, angiogenesis, and H3K4 methylation, leading to arrested EMT and lesion growth.

(Continued on next page)

\footnotetext{
* Correspondence: hoxa10@outlook.com

${ }^{\dagger}$ Equal contributors

${ }^{2}$ Shanghai Obstetrics and Gynecology Hospital, Fudan University,

419 Fangxie Road, Shanghai 200011, China

${ }^{3}$ Shanghai Key Laboratory of Female Reproductive Endocrine-Related

Diseases, Fudan University, Shanghai, China

Full list of author information is available at the end of the article
} 
(Continued from previous page)

Conclusion: In light of our previously reported reduced cellular proliferation, cell cycle progression and invasiveness resulting from the LSD1 inhibition in in vitro studies, our data strongly suggest that LSD1 is a promising therapeutic target for endometriosis.

Trial registration: Not applicable.

Keywords: Endometriosis, Epithelial-mesenchymal transition, Generalized hyperalgesia, Hotplate latency, Lysine-specific demethylase 1, Mouse, Tranylcypromine

\section{Background}

Endometriosis, characterized by the deposition and growth of functional endometrial-like tissues outside the uterine cavity, is an estrogen-dependent disease, affecting roughly $6-10 \%$ of women of reproductive age [1]. It is a leading cause of disability in women of reproductive age and a major contributing cause for dysmenorrhea, pelvic pain and subfertility [2], impacting negatively on their quality of life [3]. Despite extensive research, the development of effective and safe drugs for endometriosis treatment has been frustratingly slow [4].

Endometriosis is, first and foremost, a hormonal disease, characterized by the increased local production of estrogens due to molecular aberrations in steroidogenesis [5]. It is also recognized as a inflammatory condition [6], featuring overexpression of inflammatory genes, the release of pro-inflammatory cytokines $[7,8]$, the activation of NF-kB [9-11], and the infiltration of macrophages and lymphocytes [12-14].

Since the report, published over a decade ago, of HOXA10 hypermethylation in eutopic endometrium from women with endometriosis, which ventured the view that "endometriosis may also be an epigenetic disease" [15], accumulating evidence provides strong support for this view [16-19]. Encouragingly, drugs (the so-called "epi-drugs") targeting aberrant but reversible epigenetic modifications, mainly DNA methylation and histone deacetylation, have been shown to be of therapeutic potential for treating endometriosis. Specifically, histone deacetylases (HDACs) and DNA methyltransferases (DNMTs) have been shown to be promising targets by numerous in vitro studies [20-24]. In contrast, only a few studies have shown promising effects of HDAC inhibition in preclinical studies in endometriosis [25-27].

Epigenetic regulation of gene transcription is mediated by a diverse family of protein complexes, including chromatin remodeling and transcription factors [28]. The epigenetic landscape is complex, encompassing DNA methylation, histone code, non-coding RNA, nucleosome positioning, and DNA sequence. In particular, histone lysine methylation has recently been emerged as one key determinant of transcriptional regulation
[29]. The first protein showing histone lysine (K) demethylase (KDM) activity identified in mammals is lysine-specific demethylase 1 (LSD1 or KDM1A) [30]. LSD1 is a flavin-containing amino oxidase (AO) that specifically demethylates mono- or di-methylated lysine 4 at histone H3 (H3K4me1 and H3K4me2) via a flavin adenine dinucleotide (FAD)-monoamine oxidase (MAO) mechanism [31], and, as such, can be inhibited by compounds related to the MAO inhibitor (MAOI) class of pharmaceuticals [32]. LSD1 is intimately involved in DNA methylation [33], interact with nuclear hormone receptors such as estrogen receptor [34], have a potential role in the repression of E-cadherin and thus in the epithelialmesenchymal transition (EMT) [35] and in the regulation of VEGF [36]. It also interacts closely with HDACs to suppress gene expression [30]. In endometriosis, several lines of evidence implicate the involvement of LSD1. First, a global H3K4 and H3K9 hypomethylation in endometriotic lesions has been reported recently [37]. In addition, global $\mathrm{H} 3$ hypoacetylation as well as HDAC1 overexpression also have been reported $[17,38,39]$. While selective inhibition of prostaglandin E2 receptors EP2 and EP4 is shown to have many desirable therapeutic effects [40], it has no effect on LSD1 expression in endometriotic lesions [41].

We have recently shown that LSD1 gene and protein expression is elevated in endometriosis, and treatment of endometriotic stromal cells with tranylcypromine (TC), an LSD1 inhibitor, significantly reduced cellular proliferation, cell cycle progression, and invasiveness [42]. However, the in vivo effect of TC treatment is currently lacking. In light of scant, if any, preclinical studies on the use of epi-drugs other than HDAC inhibitors in endometriosis, this study sought to evaluate the effect of $\mathrm{TC}$ in a mouse model of endometriosis.

\section{Methods}

\section{Mouse experiment}

Forty-seven virgin female C57BL/6 mice, 8 weeks old and about $18-20 \mathrm{~g}$ in weight, were purchased from Shanghai BiKai Laboratory Animal Center (Shanghai, China) and used for this study. They were housed individually in cages, maintained under controlled conditions with a light/dark cycle of $12 / 12 \mathrm{~h}$, and had access 
to chows and water ad libitum. All experiments in this study were performed under the guidelines of the National Research Council's Guide for the Care and Use of Laboratory Animals and approved by the institutional experimental animals review board of Shanghai OB/GYN Hospital, Fudan University.

After 3 days of acclimatization and before the surgery (see Surgical procedures below), a baseline hotplate test (Test 1) was administrated to all mice as reported previously [25]. Thereafter, all mice, except 7 and 9 that were randomly selected to form groups $\mathrm{M}$ (sham surgery) and $\mathrm{S}$ (for specificity control), respectively, received an endometriosis-inducing surgery. Two weeks after the surgery, the 31 mice that received surgery were further divided at random into 3 groups: 1) untreated (U) group $(n=11)$; 2$)$ low-dose TC (L) group $(n=10)$; $3)$ high-dose TC $(\mathrm{H})$ group $(n=10)$. Mice in group $\mathrm{M}$ received similar care as others but received a sham surgery (fragments of fat tissues, instead of endometrial tissue fragments). The set-up of group $\mathrm{M}$ was meant to validate the results obtained from humans, and their endometrial tissue samples were subjected to immunohistochemistry analysis of LSD1, using the same LSD1 primary antibody used in humans. They were sacrificed 2 weeks after surgery.

Two weeks after induction, mice in group $U$ received $0.3 \mathrm{~mL}$ intra-peritoneal (i.p.) injection of sodium sulfate solution (a solvent for TC) every two days, while mice in groups $\mathrm{L}$ and $\mathrm{H}$ received i.p. injection of 1 or $2 \mathrm{mg} / \mathrm{kg}$ bodyweight TC dissolved in $0.3 \mathrm{ml}$ sodium sulfate solution every two days, respectively. Meanwhile, group S received a high-dose TC treatment in identical fashion as to group $\mathrm{H}$. The dose in group $\mathrm{H}$ was about the half of the dose used in a cancer study [43]. Group S was set up mainly to ensure that the reduced generalized hyperalgesia, if any, in mice treated with TC is not due to any possible analgesic effect of TC, but rather resulting from the treatment effect specific to endometriosis. All groups received identical care except the administration of different types and/or doses of drugs. The schematic illustration of the experimental design is shown in Fig. 1.

To gauge the change in thermal latency as a result of induced endometriosis, a second round of hotplate test (Test 2) was administrated to all mice two weeks after surgery and before the treatment. Two weeks after treatment, the third round of hotplate test (Test 3) was administrated to all mice. Then the mice were sacrificed through cervical dislocation. The abdominal cavity was immediately reopened through the original incision, and all lesions were measured with two perpendicular diameters (the length $D_{1}$ and the width $D_{2}$ ). The surface area of each endometrial implant tissue was calculated by the formula: $S=\pi \times D_{1} \times D_{2} / 4$ (in $\mathrm{mm}^{2}$ ). The total surface area of ectopic lesions in each mouse was evaluated. Ectopic lesion tissues in groups $\mathrm{H}, \mathrm{L}$ and $\mathrm{U}$ were harvested and fixed immediately after collection in $10 \%$ formalin and embedded in paraffin for histopathologic examination and immunohistochemical analysis.

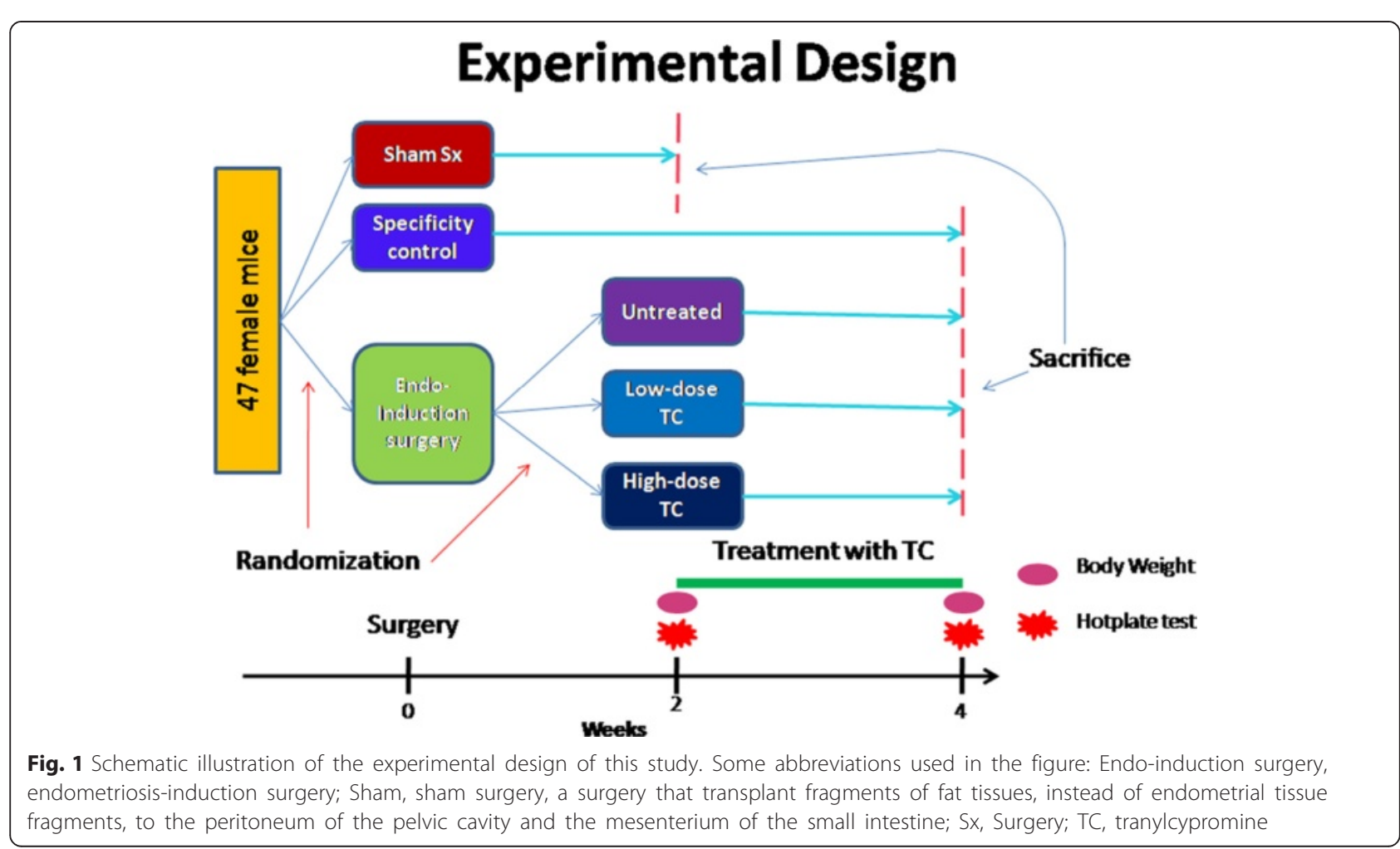




\section{Surgical procedures}

Anaesthetized with $300 \mathrm{mg} / \mathrm{kg}$ chloral hydrate, surgery was performed aseptically to auto-transplant small pieces of uterine to the peritoneum of the pelvic cavity and the mesenterium of the small intestine, as described previously [25]. Laparotomy was performed and the left uterine horn was removed. The excised horns were immersed in a sterile lactate solution and opened longitudinally. Each uterine segment was then cut into four smaller, roughly equal-sized ( $2.5 \mathrm{~mm}$ in diameter) fragments. A total of 4 uterine fragments were sutured to the peritoneal wall of the lower part of the lateral abdominal and pelvic cavity with a 7/0 braided silk suture. Then the midline incision was closed with a $3 / 0$ braided silk suture. After surgery, all mice were administrated with $40,000 \mathrm{U}$ of penicillin i.m. to prevent infection. In addition, they received subcutaneous injection of $0.2 \mathrm{mg} / \mathrm{kg}$ bodyweight $17 \beta$-estradiol solution twice a week for the next 2 weeks to stimulate and maintain the growth of endometrial implants [25]. Endometriosis was successfully induced in all mice.

\section{Immunohistochemistry and histochemistry}

Mice ectopic lesions were fixed for $24 \mathrm{~h}$ at room temperature with $10 \%$ formalin neutral buffer solution. After fixation, the tissues were placed in $70 \%$ ethanol overnight at $4{ }^{\circ} \mathrm{C}$, then embedded in paraffin and sectioned at $4-\mu \mathrm{m}$ thickness. For each paraffin-embedded tissue block, the first resultant slide was stained for $\mathrm{H} \& \mathrm{E}$ to confirm pathologic diagnosis, and the subsequent slides were stained for proliferating cell nuclear antigen (PCNA) (a marker for cellular proliferation), vascular endothelial growth factor (VEGF), CD31 (for counting microvessel density or MVD), H3K4me1, H3K4me2, E-cadherin and vimentin. Routine deparaffinization and rehydration procedures were performed following published protocols.

For antigen retrieval, the slides were heated at $98{ }^{\circ} \mathrm{C}$ in the EDTA buffer $(\mathrm{pH}$ 8.0) or the citric acid solution (pH 6.0) (depending on the primary antibody used) for a total of $30 \mathrm{~min}$ and cooled naturally at room temperature. The rabbit polyclonal antibodies against PCNA (Thermo Littleton, CO, USA), H3K4me1 (Abcam, Cambridge, MA, USA), H3K4me2 (Abcam), VEGF (Santa Cruz, TX, USA), CD31 (Abcam), E-cadherin (CST, MA, USA), vimentin (Abcam) diluted to 1:100, 1:100, 1:100, 1:50, 1:50, 1:100 and 1:200, respectively, were used as primary antibodies. Normal control mouse endometrial tissues in group $M$ and the ectopic endometrial tissues in group $\mathrm{U}$ were also incubated with a rabbit polyclonal antibody against LSD1 (1:100; Novus Biologicals, Littleton, CO,USA) at $4{ }^{\circ} \mathrm{C}$ overnight. After slides were rinsed by PBS, they were incubated at room temperature for $15 \mathrm{~min}$ and $10 \mathrm{~min}$, respectively, in biotinylated secondary antibody and treptavidin-labeled HRP (MR-SPR120, Shanghai MingRui BioTech, Shanghai, China). Finally, the sections were stained for $2 \mathrm{~min}$ or until appropriate for microscopic examination with diaminobenzidine. The sections were then counter-stained with haematoxylin, dehydrated in a graded alcohol series, cleared in xylene, and finally mounted in balsam. Human invasive breast cancer tissue samples were used as positive control. Negative control sections were treated identically, except that the specific antibody was replaced by the same concentration of normal rabbit serum. No positive reaction was observed under these conditions. Since TC blocks LSD1 activity [44], immunohistochemistry analysis of LSD1 in treated mice was not performed.

The number and intensity of positive cells were counted by Image Pro-Plus 6.0 (Media Cybernetics, Inc., Bethesda, Maryland, USA). Images were obtained with the microscope (Olympus BX51, Olympus, Tokyo, Japan) fitted with a digital camera (Olympus DP70, Olympus, Tokyo, Japan). A series of 3-5 random images on several sections were taken for each immunostained parameter to obtain a mean value. Staining was defined via color intensity, and a color mask was made. The mask was then applied equally to all images, and measurement readings were obtained. Immunohistochemical parameters assessed in the area detected included (a) integrated optical density (IOD); (b) total stained area (S); and (c) mean optical density (MOD), which is defined as $\mathrm{MOD}=\mathrm{IOD} / \mathrm{S}$, equivalent to the intensity of stain in all positive cells.

\section{Statistical analysis}

The comparison of distributions of continuous variables between or among two or more groups was made using the Wilcoxon's and Kruskal's test, respectively, and the paired Wilcoxon test was used when the before-after comparison was made for the same group of subjects. Pearson's or Spearman's rank correlation coefficient was used when evaluating correlations between two variables when both variables were continuous or when at least one variable was ordinal. To see whether TC treatment and other possible factors were responsible for the change in hotplate latency before and after the treatment, a multiple linear regression model was used. The treatment of $0,1 \mathrm{mg} / \mathrm{kg}$, and $2 \mathrm{mg} / \mathrm{kg}$ TC was coded as 0,1 , and 2 , respectively.

To further characterize the treatment effect, we carried out a hierarchical cluster analysis with scaled data and the Euclidean distance as the similarity metric, with the average linkage being the clustering method. The resulting dendrogram was represented as a heatmap. A multidimensional scaling (MDS) analysis was performed to discriminate all mice used in this study. 
$P$ values of less than 0.05 were considered statistically significant. All computations were made with R 3.2.2 [45] (www.r-project.org).

\section{Results}

Before the treatment was started, two mice in group $\mathrm{L}$ died at the 7 th day after surgery following the $17 \beta$ estradiol injection, and 2 mice each in groups $S$ and $\mathrm{H}$ were found dead at the 5 th day after the treatment was started. An autopsy ensued for all dead mice, but no apparent abscess, hemorrhage, obstruction, or embolus was found. The respiratory, circulation, and urinary systems, and the liver and spleen all appeared normal. Since mice in groups $\mathrm{H}$ and $\mathrm{S}$ all received higher TC dosage, it is likely that the toxicity might have resulted in the demise. Consequently, groups $M$, $\mathrm{U}, \mathrm{L}, \mathrm{H}$ and $\mathrm{S}$ had $7,11,8,8$ and 7 mice, respectively at the end of the experiment and were used in the following analyses. In the $\mathrm{L}$ group, $\mathrm{TC}$ appeared to be well tolerated, and no adverse event was observed.
Inhibition of LSD1 results in reduced lesion size and improved hyperalgesia in mice with induced endometriosis We first show that, compared with the control endometrium from mice that received sham surgery (and thus had no endometriosis), the LSD1 staining levels in endometriotic lesions in group $U$ mice were significantly elevated ( $p=4.4 \times 10^{-4}$; Fig. 2$)$, similar to what we found in humans [42].

We also found that TC treatment dose-dependently reduced the lesion size (Fig. 3a; $p=1.8 \times 10^{-7}, R^{2}=0.67$, by a multiple linear regression analysis with log-transformed lesion size). In fact, low- and high-dose TC treatment resulted in an average of 62.4 and $76.7 \%$ reduction in lesion size, respectively $(p=0.0013$ and $p=0.0003$, respectively; Fig. 3a).

As expected, there was no difference in hotplate latency prior to the induction of endometriosis $(p=0.16$; Fig. $2 \mathrm{~b})$. However, 2 weeks after the induction but before the TC treatment, a significant difference in hotplate latency among the 5 groups of mice was found $(p=0.0001$;

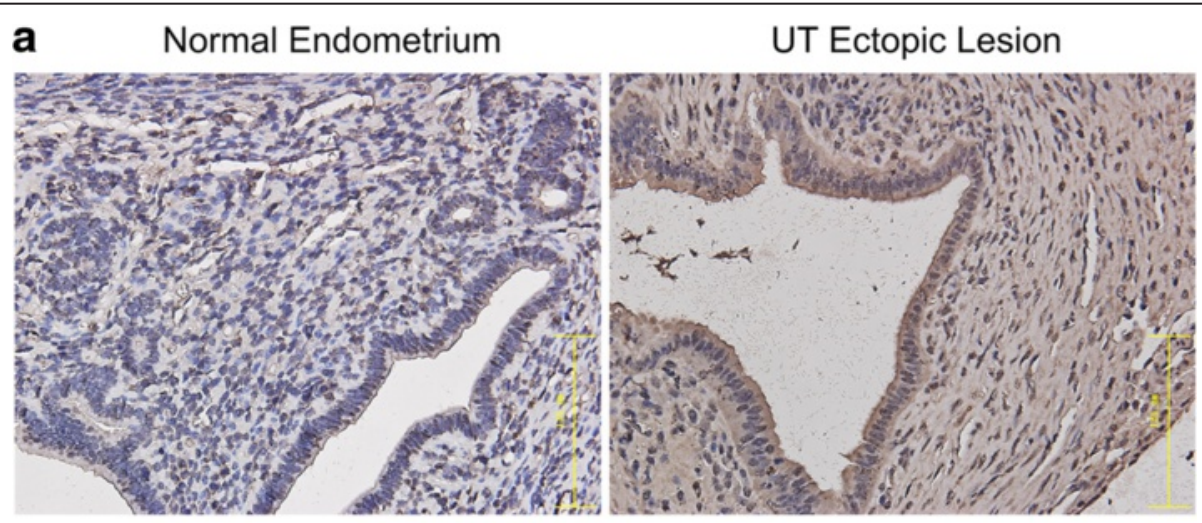

b

LSD1 staining levels in ectopic and control endometrium

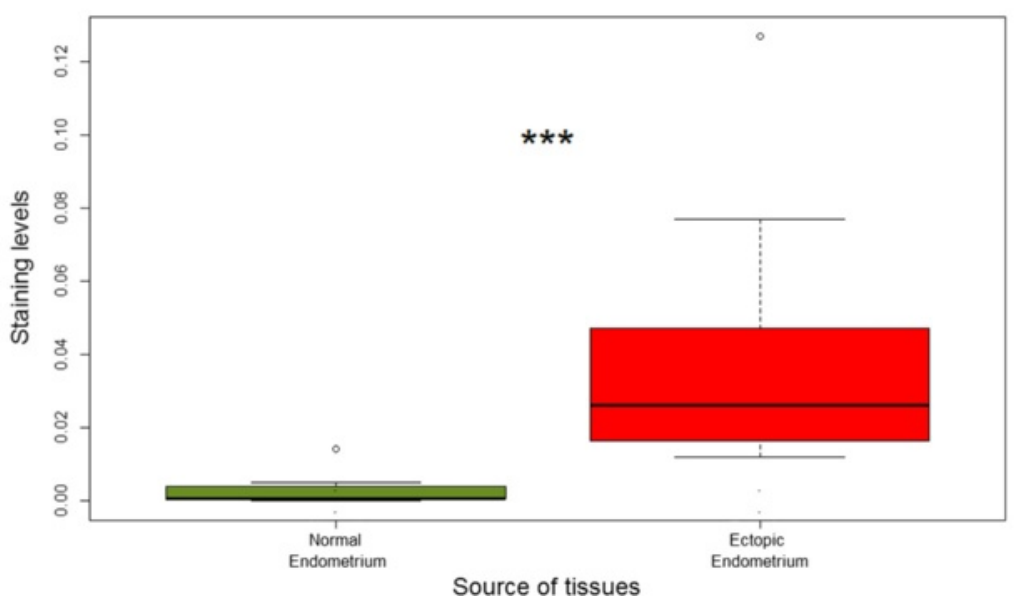

Fig. 2 a Representative photo micrographs showing immunoreactivity to LSD1 in the endometrium from a mouse that received a sham surgery and from a mouse that had induced endometriosis but received no treatment. Magnification: X400. The scale bar represents $125 \mu \mathrm{m}$. $\mathbf{b}$ Boxplot of LSD1 staining levels in normal endometrium from mice in group SHAM and in ectopic endometrium from mice in group $U$. "***" denotes the $p$-value of the difference between the two groups is less than 0.001 

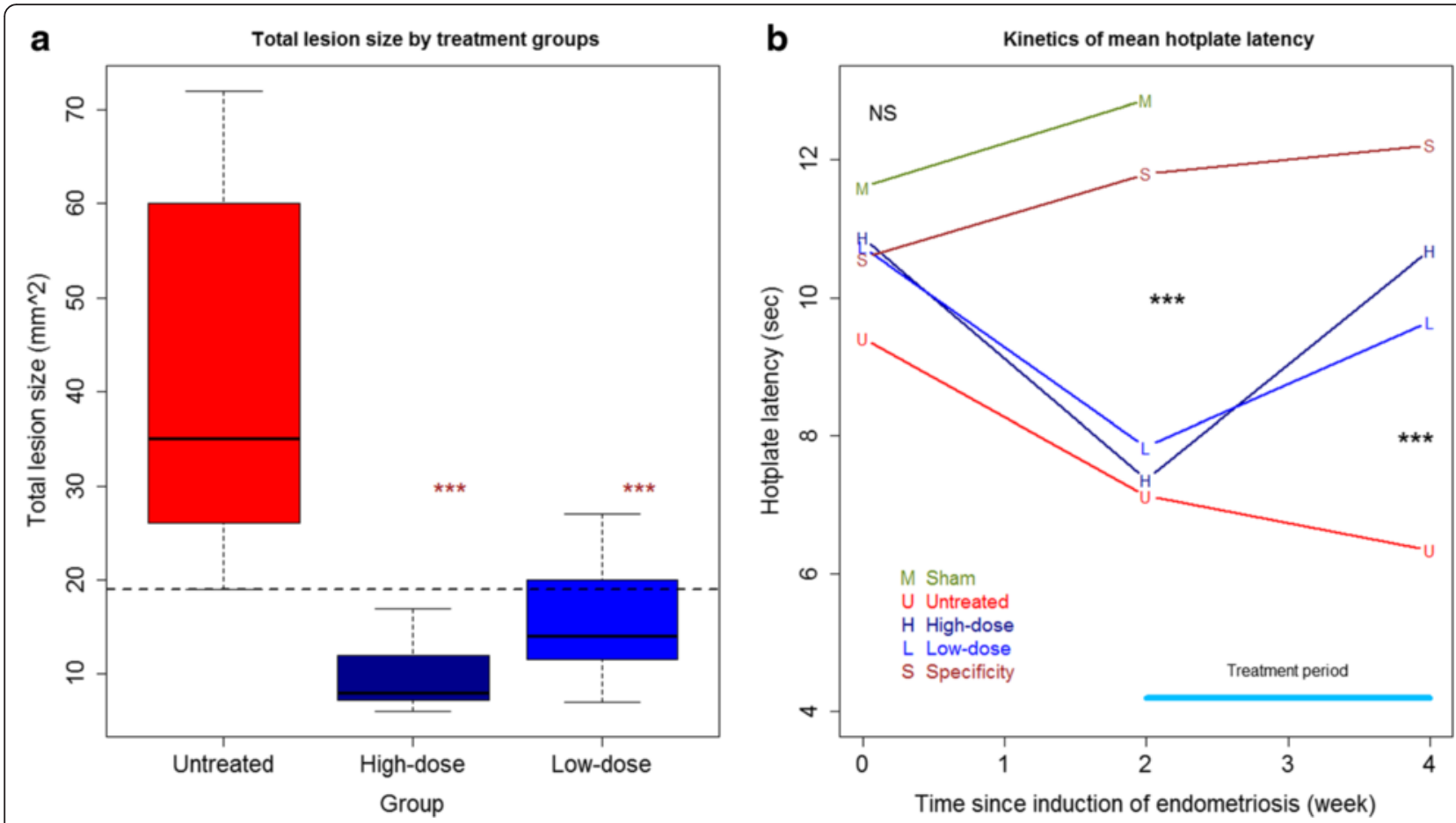

Fig. 3 a Boxplot of lesion size in different treatment groups. "***" denotes the p-values of the difference between the designated group and the untreated group are less than 0.001 . b The time-course of the mean hotplate latency in different treatment groups. Abbreviations used in the figure: $\mathrm{M}$, sham surgery group; $\mathrm{U}$, untreated mice; $\mathrm{L}$, mice treated with low-dose TC; $\mathrm{H}$, mice treated with high-dose TC; $\mathrm{S}$, specificity control group

Fig. 3b). In fact, mice with induced endometriosis had a significantly decreased latency as compared with those without $\left(p=2.1 \times 10^{-6}\right.$; Fig. $\left.3 \mathrm{~b}\right)$, consistent with what we reported previously [25-27]. Two weeks after treatment, there was a significant difference in hotplate latency among the 4 groups of mice $(p=0.0003$; Fig. 3b; Hotplate latency in the sham group was not measured). In fact, mice with endometriosis that received TC treatment had significantly longer latency-in a dose-dependent manner-than those without $(p=0.0003$, by multiple linear regression controlling for before-treatment levels and the bodyweight at sacrifice, $R^{2}=0.87$; Fig. $3 \mathrm{~b}$ ). This can be seen from the improved latency in mice with endometriosis and treated with TC but not in untreated mice (Fig. 3b). The mice in group $S$, on the hand, had no significant change in latency $(p=0.40$; Fig. $3 \mathrm{~b})$, suggesting that the improvement in hotplate latency in TC-treated mice was endometriosis-specific.

\section{Inhibition of LSD1 results in reduced proliferation and angiogenesis in ectopic endometrium}

We further performed an immunohistochemistry analysis of PNCA, VEGF, CD31, H3K4me1, H3K4me2, Ecadherin, and vimentin for ectopic endometrium. We found that PCNA, H3K4me1 and H3K4me2 staining was seen in cellular nuclei in both the stromal and epithelial cells of the ectopic endometrium, while VEGF immunoreactivity was seen mostly in the cytoplasm of glandular epithelial cells as well as of vascular endothelial cells (Fig. 4). As expected, CD31 staining was seen mostly in vascular endothelial cells, and E-cadherin staining was seen mostly in cell membranes of glandular epithelium. On the other hand, vimentin staining was seen in the cytoplasm of stromal cells (Fig. 4).

We found that the TC dosage correlated closely with the immunoreactivity to all these proteins. In particular, the TC dosage correlated negatively with staining of PCNA $\left(r=-0.87, p=2.7 \times 10^{-9}\right.$; Spearman's correlation), $\operatorname{VEGF}\left(r=-0.75, p=7.9 \times 10^{-6}\right)$, MVD (CD31) $(r=-0.80$, $\left.p=4.3 \times 10^{-7}\right)$, and vimentin $(r=-0.52, p=0.0054)$, but positively with that of E-cadherin $(r=0.55, p=0.0029)$, H3K4me1 $(r=0.64, p=0.0004)$, and H3K4me2 $(r=0.63$, $p=0.0005)$. For all markers, there was a significant difference in immunoreactivity among the 3 groups (all $p$-values $<0.015$; Fig. 5a, c-h). Precisely as expected, TC treatment resulted in elevated H3K4me1 and H3K4me2 staining levels (Fig. 5g, h). Linear regression analyses indicated that TC dosage was negatively associated with the decrease in immunoreactivity to PCNA ( $p=2.8 \times 10^{-7}, R^{2}=0.76$; square-root transformed to improve normality; Fig. $5 \mathrm{a})$, VEGF $\left(p=5.2 \times 10^{-6}, R^{2}=0.57\right.$; Fig. $5 \mathrm{c}), \operatorname{MVD}\left(p=1.5 \times 10^{-6}, R^{2}=0.61\right.$; Fig. $\left.5 \mathrm{~d}\right)$, and vimentin $\left(p=0.0026, R^{2}=0.31\right.$; Fig. 5 f) but positively 


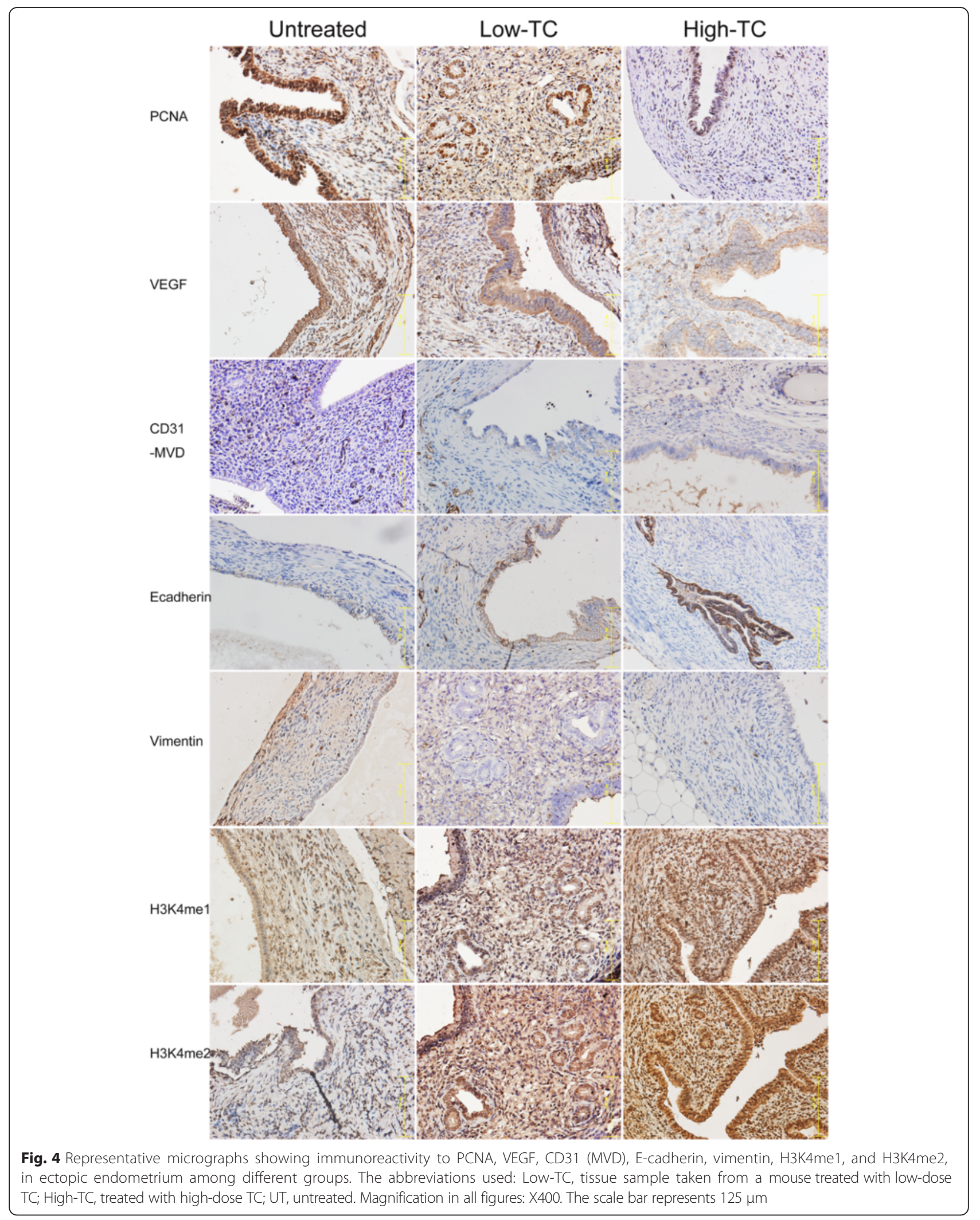




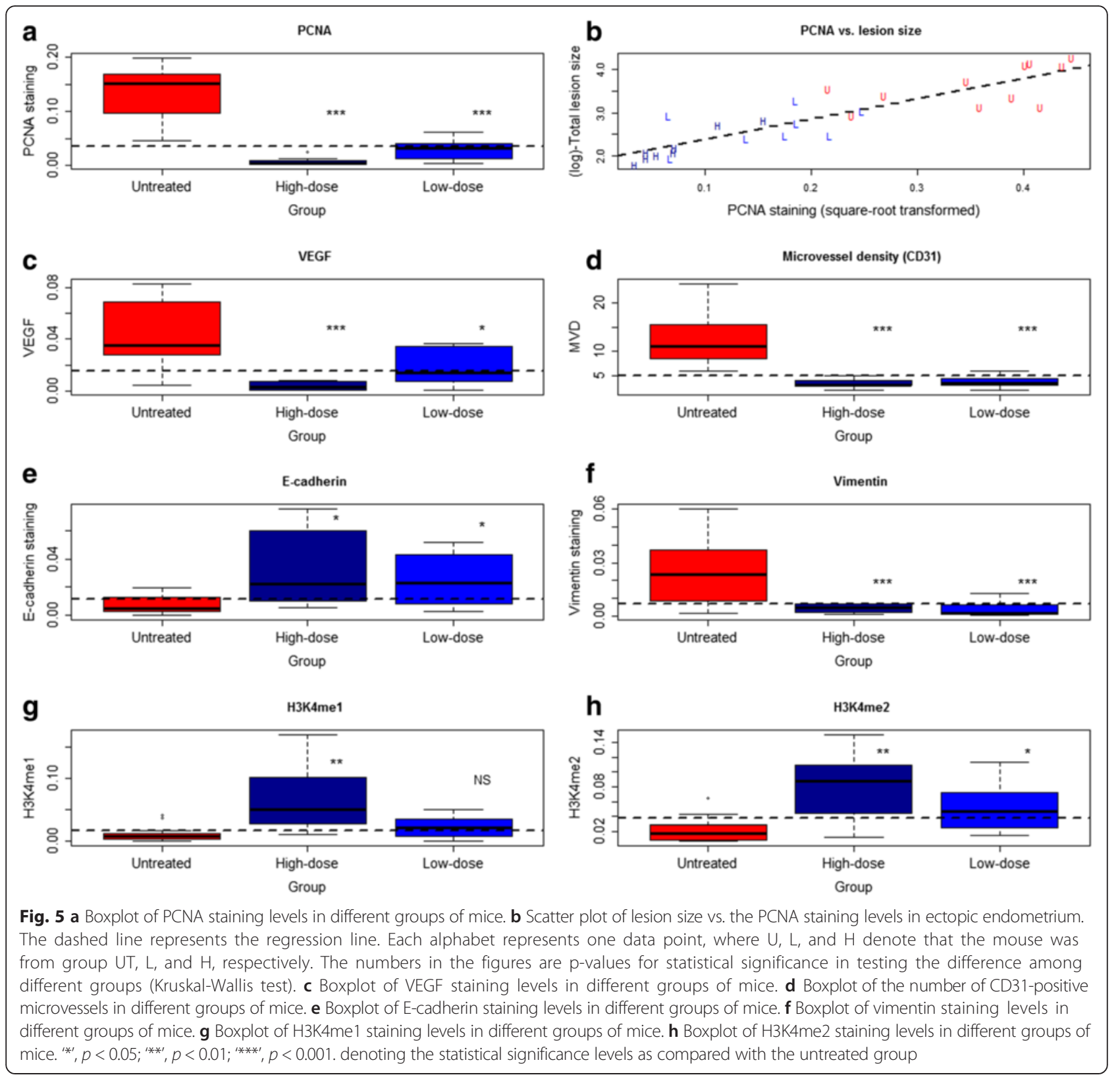

associated the increase in immunoreactivity to E-cadherin ( $p=0.0024, R^{2}=0.31$; Fig. 5e), H3K4me1 $\left(p=0.0005, R^{2}=\right.$ 0.39; Fig. 3g), and H3K4me2 ( $p=0.0004, R^{2}=0.39$; Fig. 5h).

As expected, the lesion size correlated positively with the PCNA staining levels $\left(r=0.87, p=3.5 \times 10^{-9}\right.$, both square-root transformed; Fig. 5b). The PCNA immunoreactivity correlated positively with the MVD $(r=0.64, p=0.0003)$, which, in turn, positively correlated with that of VEGF $(r=0.67, p=0.0001)$. The VEGF staining levels correlated negatively with that of both H3K4me1 $(r=-0.42, p=0.029)$ and H3K4me2 $(r=-0.49, p=0.0089)$.
Hierarchical cluster analysis of measured phenotypes and IHC immunoreactivity

To gain more insights into the possible mechanisms of TC treatment effect, we performed a hierarchical cluster analysis of all measured phenotypes and IHC immunoreactivity measurements, all square-root transformed, and the results are presented as a heatmap (Fig. 6). We can see from Fig. 6 that all mice could be grouped roughly into 2 clusters: cluster 1 is featured by a high growth propensity, namely, larger lesion size, and elevated immunoreactivity to VEGF, PCNA, vimentin and higher MVD in the ectopic endometrium. In addition, it is 


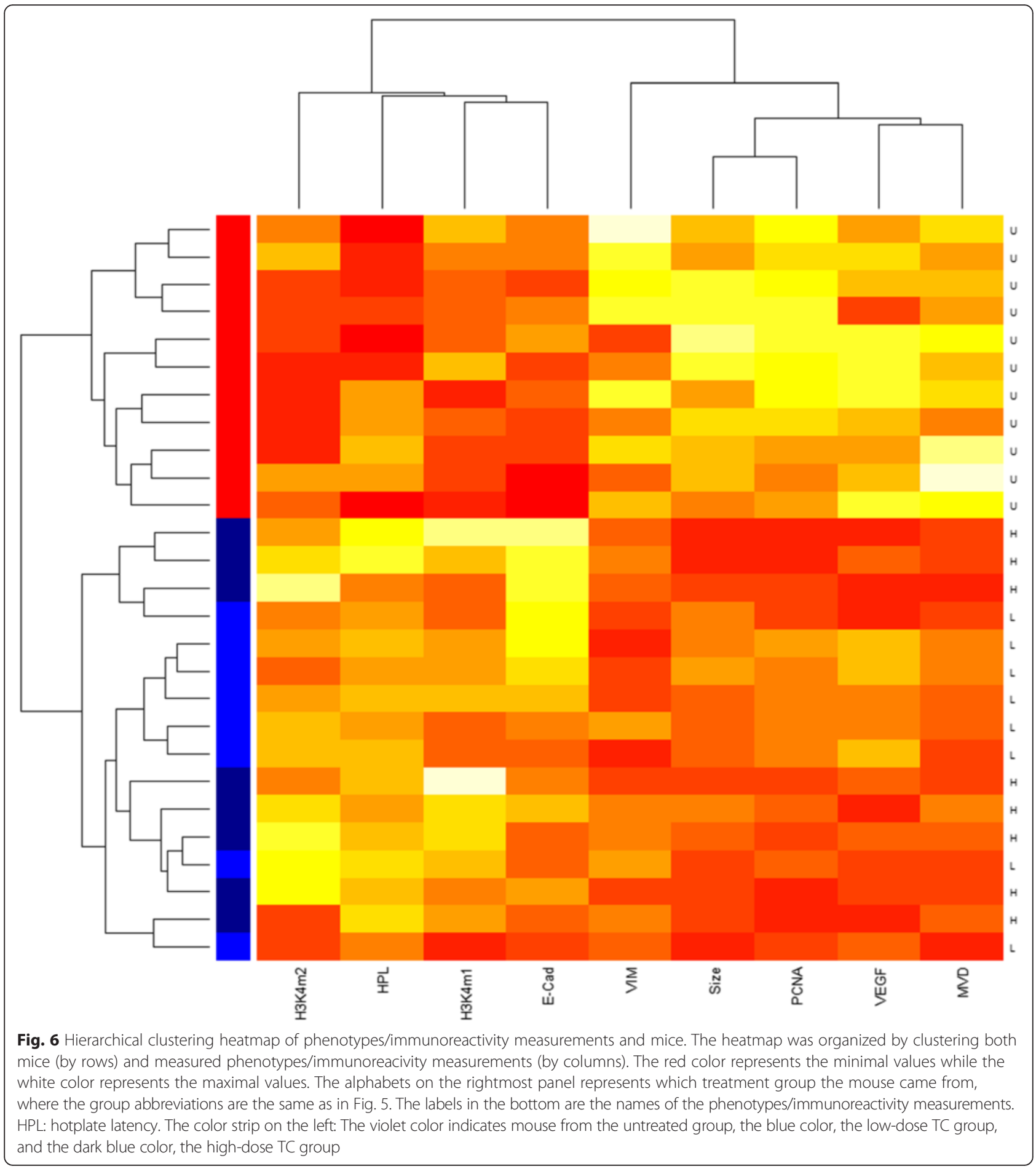

featured by shorter hotplate latency and reduced immunoreactivity to $\mathrm{H} 3 \mathrm{~K} 4 \mathrm{me} 1 / \mathrm{me} 2$ and E-cadherin. This cluster includes exclusively all mice in the untreated group. Cluster 2 is the opposite, and is characterized by a reduced growth propensity and longer latency, heavier bodyweight and elevated H3K4me1/me2 and E-cadherin staining. This cluster includes exclusively all mice that received $\mathrm{TC}$ treatment, although mice in the low- and high-dosage groups appeared to overlap (Fig. 6).

From the heatmap, it seems that those proteins involved in growth propensity can be clustered together, which forms a greater cluster with the lesion size (Fig. 6). In contrast, hotplate latency, E-cadherin and H3K4me1/me2 staining levels formed another group 
(Fig. 6). Consequently, we used these staining levels and MVD scores to carry out a multidimensional scaling analysis. The result is shown in Fig. 7. We can see that, indeed, the use of these measurements can cluster the mice very nicely, almost a perfect match with the original treatment assignment (Fig. 7).

\section{Discussion}

In this study, we have demonstrated that TC treatment significantly and substantially reduced the lesion size and improved generalized hyperalgesia in a dosedependent fashion in mice with induced endometriosis. In addition, TC treatment results in reduced immunoreactivity to VEGF, PCNA, and vimentin but elevated E-cadherin and $\mathrm{H} 3 \mathrm{~K} 4 \mathrm{me} 1 / \mathrm{me} 2$ staining levels, leading to arrested EMT and decreased MVD as well as lesion growth. This, in conjunction with our previously reported reduced cellular proliferation, cell cycle progression and invasiveness resulting from the LSD1 inhibition in vitro studies [42], strongly suggests that LSD1 is a promising therapeutic target for endometriosis.

Endometriotic epithelial cells are known to have increased invasive propensity [46, 47], which is characterized with decreased E-cadherin expression [47], likely due to its promote hypermethylation [48]. The loss of E-cadherin expression is a hallmark of EMT, a highly conserved cellular process that allows polarized and generally immotile epithelial cells to convert to motile mesenchymal cells, and occurs in embryonic development, cancer, and as a physiological response to injury [49]. EMT endows cells with migratory and invasive capabilities, increasing the invasive propensity.

EMT is now well-documented in endometriosis [50-53], featuring decreased E-cadherin expression but increased vimentin expression in the epithelial component of endometriotic lesions. Several transcription factors known to play critical roles in EMT, such as Snail, Twist and ZEB1 [49], are also reported to be upregulated in endometriosis [52]. Yet Snail uses its SNAG domain as a molecular hook to associate the LSD1/HDAC complex [54]. Once recruited to the E-cadherin promoter, the complex may modify histones to generate a repressive chromatin environment featuring H3K4 hypomethylation and $\mathrm{H} 3 / \mathrm{H} 4$ hypoacetylation [55]. Our data appear to be consistent with published data of $\mathrm{H} 3$, but not $\mathrm{H} 4$, hypoacetylation [38] and global H3K4 hypomethylation [37] and the increased LSD1 expression [42] in endometriosis.

Our data are also consistent with the abrogated EMT as a result of LSD1 inhibition through TC treatment, as seen by reduced vimentin staining but increased Ecadherin staining in the epithelial component of lesions as well as increased H3K4me1 and H3K4me2 staining levels in endometriotic lesions. The increased expression of Ecadherin, a marker of epithelial cells, and the decreased expression of vimentin, a marker of mesenchymal cells,

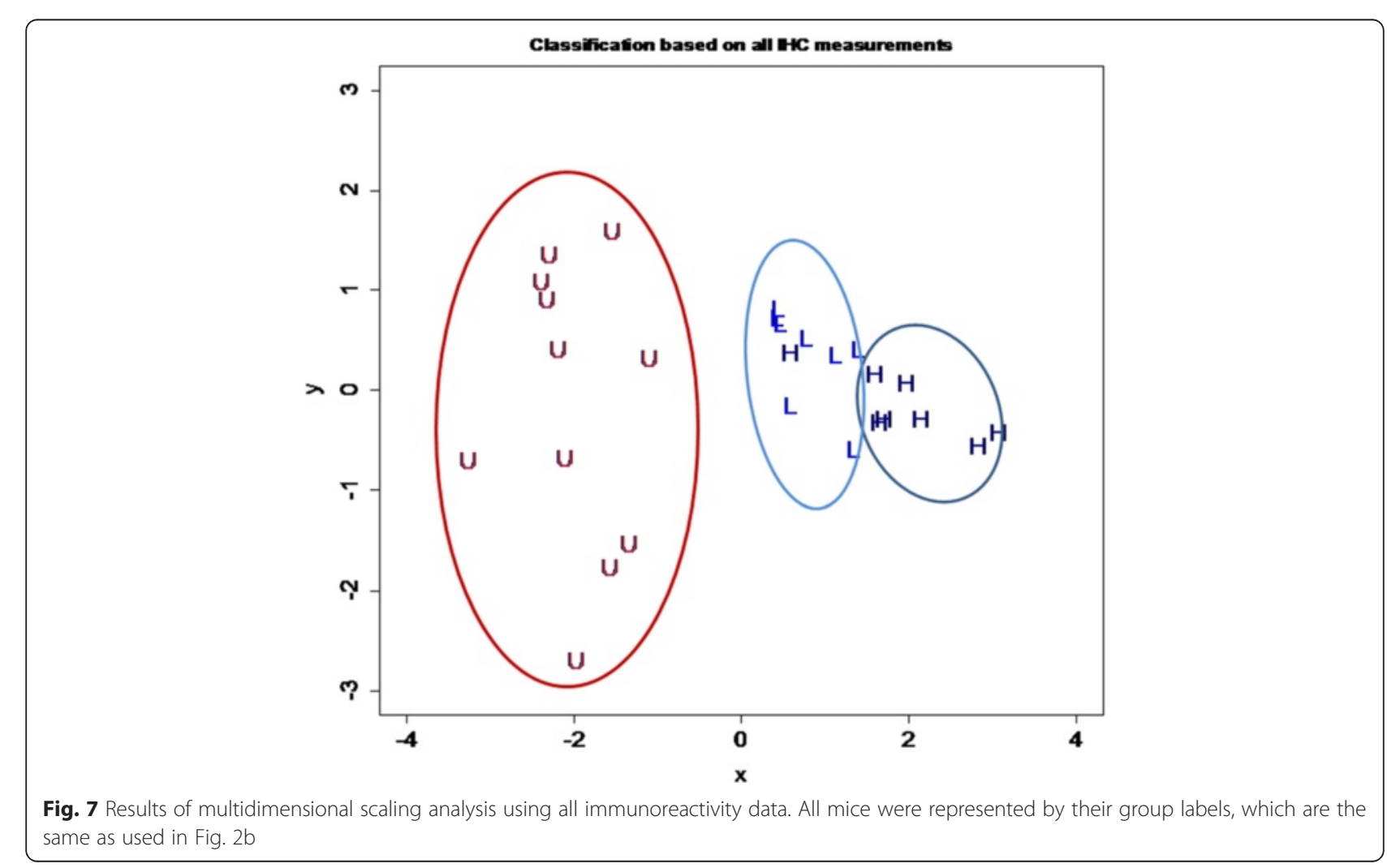


seen in mice treated with TC is consistent with reports that LSD1 is essential for Snail-mediated transcriptional repression and for maintenance of the silenced state of Snai1 target genes, including E-cadherin, in invasive cancer cells [35], and that chromatin reprogramming during EMT is LSD1-dependent [56]. Inhibition by TC also is found to suppress Slug-LSD1 interaction, blocking cancer cell motility and invasion [57]. Since loss of E-cadherin is intimately related with EMT and invasiveness, even in endometriosis $[48,51]$, these results appear to give more credence to the reduced invasiveness of endometriotic stromal cells treated with TC (Fig. 5).

The elevated LSD1 staining levels in ectopic implants as compared with normal endometrium are also consistent with our human data [42]. The reduced VEGF expression is consistent with the report that functional deletion of LSD1 impairs VEGF transcription [36], and also is consistent with the reduced MVD, and subsequently PCNA staining levels and lesion size.

Our data demonstrate a potent therapeutic effect of LSD1 inhibition by TC, as evidenced by close to $2 / 3$ reduction in lesion size in low-dose group and over $3 / 4$ of reduction in high-dose group. Aside from the abrogation of the EMT process and thus invasiveness in the development of endometriosis, the decreased angiogenesis and cellular proliferation, LSD1 suppression by TC may also have other desirable therapeutic effects, yet to be identified, since the H3K4 and H3K9 demethylation is likely to be global. For example, as a nonselective and irreversible MAO inhibitor, TC is anti-depressant and anxiolytic (used only in Germany). After all, women with endometriosis, especially those with dysmenorrhea and infertility, may experience anxiety [58]. It is also possible that its use, in combination with other therapeutics such as HDAC inhibitors, may generate synergistic therapeutic effect as in acute myeloid leukemia [59].

Given the cross-talks between LSD1 and HDACs $[30,60]$, it is not surprising to see that the use of LSD1 inhibitors may sensitize cancer cells to HDAC inhibitors [61]. HDAC inhibition also has been shown to stimulate H3K4 methylation through suppression of KDMs including LSD1 [62]. This, in conjunction with the data presented here and elsewhere, suggests that LSD1 is promising candidate for targeted therapy to treat endometriosis.

However, caution also should be exercised. Endometriosis is largely a benign disease and certainly is not lifethreatening. As such, it places higher premium on drug safety as compared with other life-threatening diseases such as cancer. While LSD1 suppression by TC may hold promises in treating endometriosis, the high mortality in mice that received high-dose of TC raises a red flag. It is also conceivable that H3K4 demethylation may be part of the normal physiology, and, as such, TC treatment may have off-target toxicity. Whether TC should be used in treating human endometriosis, dosage, formulation, and route of delivery surely will need more investigation and a thorough evaluation of the benefit versus risk ratio. The encouraging results shown in this study merely demonstrate the possibility of targeting LSD1 for therapeutic purpose. They are not meant to advocate TC per se for the treatment of human endometriosis. Encouragingly, several LSD1 inhibitors are on the horizon [63].

\section{Conclusions}

In summary, we found that tranylcypromine treatment results in marked changes that are consistent with retarded EMT, decreased angiogenesis and lesion growth, and improved hyperalgesia in mice with induced endometriosis, along with increased H3K4 methylation. Taken together, these results suggest that LSD1 may be a promising therapeutic target for endometriosis.

\section{Abbreviations \\ AO: amino oxidase; DNMT: methyltransferase; EMT: epithelial-mesenchymal transition; FAD: flavin adenine dinucleotide; H3K4me1: mono-methylated lysine 4 at histone $\mathrm{H} 3$; $\mathrm{H} 3 \mathrm{~K} 4 \mathrm{me} 2$ : di-methylated lysine 4 at histone $\mathrm{H3}$; HDAC: histone deacetylase; LSD1: Iysine-specific demethylase 1; MAO: monoamine oxidase; MDS: multidimensional scaling; MVD: microvessel density; PCNA: proliferating cell nuclear antigen; TC: tranylcypromine; VEGF: vascular endothelial growth factor.}

\section{Competing interests}

The authors declare that they have no competing interests.

\section{Authors' contributions}

SWG was responsible for the design of the experiment, data analysis and drafting the manuscript. QYS managed the project and contributed to manuscript preparation, DD and XSL carried out the experiment and IHC analysis. All authors read and approved the final manuscript.

\section{Acknowledgment}

This research was supported in part by grant $2011 \mathrm{KYA} 146$ (QYS) from the Bureau of Health and Family Planning of Zhejiang Province, grants 81270676 (SWG), 81471434 (SWG), 81530040 (SWG), 81070470 (XSL), 81370695 (XSL) and 81401183 (DD) from the National Natural Science Foundation of China, and grant $2013 Z$ YJB0019 (XSL) from Shanghai Municipal Commission of Health and Family Planning.

\section{Grant support}

This research was supported in part by grant $2011 \mathrm{KYA} 146$ (QYS) from the Bureau of Health and Family Planning of Zhejiang Province, grants 81270676 (SWG), 81471434 (SWG), 81530040 (SWG), 81370695 (XSL) and 81401183 (DD) from the National Natural Science Foundation of China, and grant $2013 Z$ YJB0019 (XSL) from Shanghai Municipal Commission of Health and Family Planning.

\section{Author details}

${ }^{1}$ Cixi Child and Maternal Hospital, 1288 Er'Zhaotan Road, Baishalu, Cixi, Zhejiang, China. ${ }^{2}$ Shanghai Obstetrics and Gynecology Hospital, Fudan University,

419 Fangxie Road, Shanghai 200011, China. ${ }^{3}$ Shanghai Key Laboratory of Female Reproductive Endocrine-Related Diseases, Fudan University, Shanghai, China.

Received: 5 February 2016 Accepted: 5 April 2016

Published online: 09 April 2016 


\section{References}

1. Giudice LC, Kao LC. Endometriosis. Lancet. 2004;364:1789-99.

2. Farquhar CM. Extracts from the "clinical evidence". Endometriosis. BMJ. 2000; 320:1449-52.

3. Nnoaham KE, Hummelshoj L, Webster P, d'Hooghe T, de Cicco NF, de Cicco NC, et al. Impact of endometriosis on quality of life and work productivity: a multicenter study across ten countries. Fertil Steril. 2011;96:366-73. e8.

4. Vercellini $P$, Crosignani $P$, Somigliana E, Vigano P, Frattaruolo MP, Fedele L 'Waiting for Godot': a commonsense approach to the medical treatment of endometriosis. Hum Reprod. 2011;26:3-13.

5. Bulun SE, Lin Z, Imir G, Amin S, Demura M, Yilmaz B, et al. Regulation of aromatase expression in estrogen-responsive breast and uterine disease: from bench to treatment. Pharmacol Rev. 2005;57:359-83.

6. Burney RO, Giudice LC. Pathogenesis and pathophysiology of endometriosis. Fertil Steril. 2012:98:511-9.

7. Akoum A, Lemay A, Paradis I, Rheault N, Maheux R. Secretion of interleukin6 by human endometriotic cells and regulation by proinflammatory cytokines and sex steroids. Hum Reprod. 1996;11:2269-75.

8. Wu MY, Ho HN. The role of cytokines in endometriosis. Am J Reprod Immunol. 2003;49:285-96.

9. Guo SW. Nuclear factor-kappab (NF-kappaB): an unsuspected major culprit in the pathogenesis of endometriosis that is still at large? Gynecol Obstet Invest. 2007:63:71-97.

10. Gonzalez-Ramos R, Donnez J, Defrere S, Leclercq I, Squifflet J, Lousse JC, et al. Nuclear factor-kappa B is constitutively activated in peritoneal endometriosis. Mol Hum Reprod. 2007;13:503-9.

11. Gonzalez-Ramos R, Van Langendonckt A, Defrere S, Lousse JC, Colette S, Devoto $L$, et al. Involvement of the nuclear factor-kappaB pathway in the pathogenesis of endometriosis. Fertil Steril. 2010;94:1985-94.

12. Nomiyama M, Hachisuga T, Sou H, Nakamura K, Matsumoto Y, Iwasaka T, et al. Local immune response in infertile patients with minimal endometriosis. Gynecol Obstet Invest. 1997:44:32-7.

13. Khan KN, Masuzaki H, Fujishita A, Kitajima M, Sekine I, Ishimaru T. Differential macrophage infiltration in early and advanced endometriosis and adjacent peritoneum. Fertil Steril. 2004;81:652-61

14. Bacci M, Capobianco A, Monno A, Cottone L, Di Puppo F, Camisa B, et al. Macrophages are alternatively activated in patients with endometriosis and required for growth and vascularization of lesions in a mouse model of disease. Am J Pathol. 2009;175:547-56.

15. Wu Y, Halverson G, Basir Z, Strawn E, Yan P, Guo SW. Aberrant methylation at HOXA10 may be responsible for its aberrant expression in the endometrium of patients with endometriosis. Am J Obstet Gynecol. 2005;193:371-80.

16. Guo SW. Epigenetics of endometriosis. Mol Hum Reprod. 2009;15:587-607.

17. Colon-Diaz M, Baez-Vega P, Garcia M, Ruiz A, Monteiro JB, Fourquet J, et al. HDAC1 and HDAC2 are differentially expressed in endometriosis. Reprod Sci. 2012;19:483-92.

18. Naqvi H, llagan $Y$, Krikun G, Taylor HS. Altered genome-wide methylation in endometriosis. Reprod Sci. 2014;21:1237-43.

19. Dyson MT, Kakinuma T, Pavone ME, Monsivais D, Navarro A, Malpani SS, et al. Aberrant expression and localization of deoxyribonucleic acid methyltransferase 3B in endometriotic stromal cells. Fertil Steril. 2015;104:953-63. e2.

20. Wu Y, Guo SW. Inhibition of proliferation of endometrial stromal cells by trichostatin A, RU486, CDB-2914, N-acetylcysteine, and ICI 182780. Gynecol Obstet Invest. 2006:62:193-205.

21. WU Y, Guo SW. Suppression of IL-1 beta-induced COX-2 expression by trichostatin A (TSA) in human endometrial stromal cells. Eur J Obstet Gynecol Reprod Biol. 2007;135:88-93.

22. Wu Y, Guo SW. Histone deacetylase inhibitors trichostatin A and valproic acid induce cell cycle arrest and p21 expression in immortalized human endometrial stromal cells. Eur J Obstet Gynecol Reprod Biol. 2008;137:198-203.

23. Imesch $P$, Fink D, Fedier A. Romidepsin reduces histone deacetylase activity, induces acetylation of histones, inhibits proliferation, and activates apoptosis in immortalized epithelial endometriotic cells. Fertil Steril. 2010;94:2838-42.

24. Imesch P, Samartzis EP, Schneider M, Fink D, Fedier A. Inhibition of transcription, expression, and secretion of the vascular epithelial growth factor in human epithelial endometriotic cells by romidepsin. Fertil Steril. 2011;95:1579-83.

25. Lu Y, Nie J, Liu X, Zheng Y, Guo SW. Trichostatin A, a histone deacetylase inhibitor, reduces lesion growth and hyperalgesia in experimentally induced endometriosis in mice. Hum Reprod. 2010;25:1014-25.
26. Zhao T, Liu X, Zhen X, Guo SW. Levo-tetrahydropalmatine retards the growth of ectopic endometrial implants and alleviates generalized hyperalgesia in experimentally induced endometriosis in rats. Reprod Sci. 201 1;18:28-45.

27. Liu M, Liu X, Zhang Y, Guo SW. Valproic acid and progestin inhibit lesion growth and reduce hyperalgesia in experimentally induced endometriosis in rats. Reprod Sci. 2012;19:360-73.

28. Allis CD, Berger SL, Cote J, Dent S, Jenuwien T, Kouzarides T, et al. New nomenclature for chromatin-modifying enzymes. Cell. 2007;131:633-6.

29. Cheng X, Blumenthal RM. Coordinated chromatin control: structural and functional linkage of DNA and histone methylation. Biochemistry. 2010;49: 2999-3008.

30. Shi Y, Lan F, Matson C, Mulligan P, Whetstine JR, Cole PA, et al. Histone demethylation mediated by the nuclear amine oxidase homolog LSD1. Cell. 2004;119:941-53.

31. Mosammaparast N, Shi Y. Reversal of histone methylation: biochemical and molecular mechanisms of histone demethylases. Annu Rev Biochem. 2010; 79:155-79.

32. Binda C, Valente S, Romanenghi M, Pilotto S, Cirilli R, Karytinos A, et al. Biochemical, structural, and biological evaluation of tranylcypromine derivatives as inhibitors of histone demethylases LSD1 and LSD2. J Am Chem Soc. 2010:132:6827-33.

33. Wang J, Hevi S, Kurash JK, Lei H, Gay F, Bajko J, et al. The lysine demethylase LSD1 (KDM1) is required for maintenance of global DNA methylation. Nat Genet. 2009:41:125-9.

34. Perillo B, Ombra MN, Bertoni A, Cuozzo C, Sacchetti S, Sasso A, et al. DNA oxidation as triggered by H3K9me2 demethylation drives estrogen-induced gene expression. Science. 2008;319:202-6.

35. Lin T, Ponn A, Hu X, Law BK, Lu J. Requirement of the histone demethylase LSD1 in Snai1-mediated transcriptional repression during epithelialmesenchymal transition. Oncogene. 2010;29:4896-904

36. Kashyap V, Ahmad S, Nilsson EM, Helczynski L, Kenna S, Persson JL, et al. The lysine specific demethylase-1 (LSD1/KDM1A) regulates VEGF-A expression in prostate cancer. Mol Oncol. 2013;7:555-66.

37. Xiaomeng $X$, Ming $Z$, Jiezhi M, Xiaoling F. Aberrant histone acetylation and methylation levels in woman with endometriosis. Arch Gynecol Obstet. 2013:287:487-94.

38. Monteiro JB, Colon-Diaz M, Garcia M, Gutierrez S, Colon M, Seto E, et al. Endometriosis is characterized by a distinct pattern of histone 3 and histone 4 lysine modifications. Reprod Sci. 2014;21:305-18.

39. Samartzis EP, Noske A, Samartzis N, Fink D, Imesch P. The expression of histone deacetylase 1, but not other class I histone deacetylases, is significantly increased in endometriosis. Reprod Sci. 2013;20:1416-22.

40. Banu SK, Lee J, Speights Jr VO, Starzinski-Powitz A, Arosh JA. Selective inhibition of prostaglandin E2 receptors EP2 and EP4 induces apoptosis of human endometriotic cells through suppression of ERK1/2, AKT, NFkappaB, and beta-catenin pathways and activation of intrinsic apoptotic mechanisms. Mol Endocrinol. 2009;23:1291-305.

41. Arosh JA, Lee J, Starzinski-Powitz A, Banu SK. Selective inhibition of prostaglandin E2 receptors EP2 and EP4 modulates DNA methylation and histone modification machinery proteins in human endometriotic cells. Mol Cell Endocrinol. 2015:409:51-8.

42. Ding D, Liu X, Guo SW. Overexpression of lysine-specific demethylase 1 in ovarian endometriomas and its inhibition reduces cellular proliferation, cell cycle progression, and invasiveness. Fertil Steril. 2014;101:740-9.

43. Schulte JH, Lim S, Schramm A, Friedrichs N, Koster J, Versteeg R, et al. Lysine-specific demethylase 1 is strongly expressed in poorly differentiated neuroblastoma: implications for therapy. Cancer Res. 2009;69:2065-71.

44. Jie Z, Li T, Jia-Yun H, Qiu J, Ping-Yao Z, Houyan S. Trans-2phenylcyclopropylamine induces nerve cells apoptosis in zebrafish mediated by depression of LSD1 activity. Brain Res Bull. 2009;80:79-84.

45. Team. RC. R: a language and environment for statistical computing. Vienna, Austria: R Foundation for Statistical Computing; 2013.

46. Gaetje R, Kotzian S, Herrmann G, Baumann R, Starzinski-Powitz A. Invasiveness of endometriotic cells in vitro. Lancet. 1995:346:1463-4.

47. Gaetje R, Kotzian S, Herrmann G, Baumann R, Starzinski-Powitz A. Nonmalignant epithelial cells, potentially invasive in human endometriosis, lack the tumor suppressor molecule E-cadherin. Am J Pathol. 1997:150:461-7.

48. Wu Y, Starzinski-Powitz A, Guo SW. Trichostatin A, a histone deacetylase inhibitor, attenuates invasiveness and reactivates E-cadherin expression in immortalized endometriotic cells. Reprod Sci. 2007;14:374-82. 
49. Thiery JP, Acloque H, Huang RY, Nieto MA. Epithelial-mesenchymal transitions in development and disease. Cell. 2009;139:871-90.

50. Grund EM, Kagan D, Tran CA, Zeitvogel A, Starzinski-Powitz A, Nataraja S, et al. Tumor necrosis factor-alpha regulates inflammatory and mesenchymal responses via mitogen-activated protein kinase kinase, p38, and nuclear factor kappaB in human endometriotic epithelial cells. Mol Pharmacol. 2008; 73:1394-404.

51. Matsuzaki S, Darcha C. Epithelial to mesenchymal transition-like and mesenchymal to epithelial transition-like processes might be involved in the pathogenesis of pelvic endometriosis. Hum Reprod. 2012;27:712-21.

52. Bartley J, Julicher A, Hotz B, Mechsner S, Hotz H. Epithelial to mesenchymal transition (EMT) seems to be regulated differently in endometriosis and the endometrium. Arch Gynecol Obstet. 2014;289:871-81.

53. Liao CJ, Li PT, Lee YC, Li SH, Chu ST. Lipocalin 2 induces the epithelialmesenchymal transition in stressed endometrial epithelial cells: possible correlation with endometriosis development in a mouse model. Reproduction. 2014;147:179-87.

54. Lin Y, Wu Y, Li J, Dong C, Ye X, Chi Yl, et al. The SNAG domain of Snail1 functions as a molecular hook for recruiting lysine-specific demethylase 1. EMBO J. 2010;29:1803-16.

55. Lin Y, Dong C, Zhou BP. Epigenetic regulation of EMT: the Snail story. Curr Pharm Des. 2014;20:1698-705.

56. McDonald OG, Wu H, Timp W, Doi A, Feinberg AP. Genome-scale epigenetic reprogramming during epithelial-to-mesenchymal transition. Nat Struct Mol Biol. 2011;18:867-74.

57. Ferrari-Amorotti G, Fragliasso V, Esteki R, Prudente Z, Soliera AR, Cattelani S, et al. Inhibiting interactions of lysine demethylase LSD1 with snail/slug blocks cancer cell invasion. Cancer Res. 2013;73:235-45.

58. Chen LC, Hsu JW, Huang KL, Bai YM, Su TP, Li CT, et al. Risk of developing major depression and anxiety disorders among women with endometriosis: A longitudinal follow-up study. J Affect Disord. 2016;190:282-5.

59. Fiskus W, Sharma S, Shah B, Portier BP, Devaraj SG, Liu K, et al. Highly effective combination of LSD1 (KDM1A) antagonist and pan-histone deacetylase inhibitor against human AML cells. Leukemia. 2014;28:2155-64.

60. Vasilatos SN, Katz TA, Oesterreich S, Wan Y, Davidson NE, Huang Y. Crosstalk between lysine-specific demethylase 1 (LSD1) and histone deacetylases mediates antineoplastic efficacy of HDAC inhibitors in human breast cancer cells. Carcinogenesis. 2013;34:1196-207.

61. Singh MM, Manton CA, Bhat KP, Tsai WW, Aldape K, Barton MC, et al. Inhibition of LSD1 sensitizes glioblastoma cells to histone deacetylase inhibitors. Neuro Oncol. 2011;13:894-903.

62. Huang PH, Chen CH, Chou CC, Sargeant AM, Kulp SK, Teng CM, et al. Histone deacetylase inhibitors stimulate histone $\mathrm{H} 3$ lysine 4 methylation in part via transcriptional repression of histone $\mathrm{H} 3$ lysine 4 demethylases. Mol Pharmacol. 2011;79:197-206.

63. Zheng YC, Ma J, Wang Z, Li J, Jiang B, Zhou W, et al. A systematic review of histone lysine-specific demethylase 1 and its inhibitors. Med Res Rev. 2015; 35:1032-71.

\section{Submit your next manuscript to BioMed Central and we will help you at every step:}

- We accept pre-submission inquiries

- Our selector tool helps you to find the most relevant journal

- We provide round the clock customer support

- Convenient online submission

- Thorough peer review

- Inclusion in PubMed and all major indexing services

- Maximum visibility for your research

Submit your manuscript at www.biomedcentral.com/submit
Biomed Central 